\title{
Attitudes and concerns of neonatologists and nurses to family- integrated-care in neonatal intensive care units in China
}

\author{
Xiying Xiang ${ }^{1,2}$, Shiwen Xia ${ }^{3}$, Xing Zhu ${ }^{4}$, Xiangyu Gao ${ }^{5}$, Xirong Gao ${ }^{6}$, Aiming Zhang ${ }^{7}$, Shoo K. Lee ${ }^{8,9}$, \\ Mingyan Hei ${ }^{1,2}$ \\ ${ }^{1}$ Neonatal Center, Beijing Children's Hospital, Capital Medical University, Beijing, China; ${ }^{2}$ National Center for Child Health, Beijing, China; \\ ${ }^{3}$ Department of Neonatology, Hubei Province Women and Children Hospital, Wuhan, China; ${ }^{4}$ Department of Pediatrics, the Third Xiangya \\ Hospital of Central South University, Changsha, China; ${ }^{5}$ Department of Pediatrics, Southeast University Affiliated Xuzhou Hospital, Xuzhou, \\ China; ${ }^{6}$ Department of Neonatology, Hunan Children's Hospital, Changsha, China; ${ }^{7}$ Department of Pediatrics, Hunan Provincial People's Hospital, \\ Changsha, China; ${ }^{8}$ Maternal-Infant Care Research Centre, Mount Sinai Hospital, Toronto, ON, Canada; ${ }^{9}$ Department of Pediatrics, University of \\ Toronto Faculty of Medicine, Toronto, ON, Canada \\ Contributions: (I) Conception and design: M Hei, SK Lee; (II) Administrative support: None; (III) Provision of study materials or patients: X Xiang, S \\ Xia, X Gao, X Gao, A Zhang; (IV) Collection and assembly of data: X Xiang, X Gao, X Gao, A Zhang, S Xia; (V) Data analysis and interpretation: X \\ Xiang, X Gao, M Hei, SK Lee; (VI) Manuscript writing: All authors; (VII) Final approval of manuscript: All authors. \\ Correspondence to: Mingyan Hei, MD, PhD. Neonatal Center, Beijing Children's Hospital, Capital Medical University; National Center for Child \\ Health, Beijing, China. Email: heimingyan@bch.com.cn; Shoo K. Lee, MBBS, FRCPC, PhD. Department of Pediatrics, Mount Sinai Hospital, \\ Toronto, ON, M5G 1X5, Canada. Email: Shoo.Lee@sinaihealthsystem.ca.
}

Background: Parents in China are denied visitation of their newborns in neonatal intensive care unit (NICU), leading to a prolonged period of parent-infant separation. The family-integrated care (FICare) model, which supports the integration of parents into the NICU team, is gradually being introduced in China. Considering resistance to the implementation of FICare, this study aimed to assess the attitudes and concerns of neonatologists and nurses towards FICare in China.

Methods: Using a before and after study design, a qualitative analysis was conducted to determine the perceptions and attitudes of medical professionals towards FICare in China. A total of 34 neonatologists and 94 nurses from 5 tertiary NICUs in China were enrolled. A self-developed questionnaire was used. The study steps included reading session and then survey for the first time (survey 1), a FICare getting buy-in education session (4 hours), a group discussion session, and finally repeat the questionnaire (survey 2). The surveys were completed by trained researchers regarding willingness, acceptance and concerns of implementing FICare in NICUs in China. Differences in attitudes towards FICare were compared between groups (Chi-square/correction for continuity).

Results: There are positive responses in neonatologists and nurses regarding the necessity (Survey 1: 58.8\% and 57.4\%; Survey 2: 88.2\% and 67.0\%), feasibility (Survey 1: 17.6\% and 19.1\%; Survey 2: 32.3\% and $34.0 \%$ ), and interest in joining FICare (Survey 1: 82.4\% and 83.0\%; Survey 2: $97.1 \%$ and $85.1 \%$ ). A higher proportion of neonatologists indicated that FICare could promote breastfeeding in the NICU comparing to nurses (Survey 1: $47.1 \%$ vs. 19.1\%; Survey 2: $61.8 \%$ and $46.8 \%$ respectively). Most of the neonatologists and nurses are not sure whether FICare can shorten the hospital stay (Survey 1: 82.3\% and 68.1\%; Survey 2: $85.3 \%$ and $60.6 \%$ ) or improve the doctor-patient relationship (Survey 1: 58.8\% and 68.1\%; Survey 2: 73.5\% and $69.1 \%$ ). Challenges concerning the implementation of FICare were identified as inadequate ward space, lack of human resources, and potential increases in nosocomial infection.

Conclusions: The getting buy-in education program in introducing new paradigms of neonatal care may help on how to design and implement more effective educational tools for FICare.

Keywords: Attitude; concerns; family-integrated-care (FICare); neonatal intensive care unit (NICU); China

Submitted Mar 02, 2020. Accepted for publication Aug 17, 2020.

(C) Translational Pediatrics. All rights reserved. 
doi: $10.21037 /$ tp-20-60

View this article at: http://dx.doi.org/10.21037/tp-20-60

\section{Introduction}

Since the early 2000s, neonatal intensive care unit (NICU) admission rates of preterm infants in China have steadily increased $(1,2)$, but the overall model of care in Chinese NICUs has remained unchanged. Parents in China are denied visitation of their newborn in the NICU in most cases, leading to prolonged period of NICU stay and leaving the care of their infants to be provided solely by medical professionals $(3,4)$. The separation between mothers and their newborns prevents preterm infants from being breastfed in the NICU and causes maternal anxiety after delivery (5). Given that inhibition of parent-infant interaction can have negative consequences on both the infant and parents, efforts are being made to introduce family integrated care (FICare) to neonatal units in China.

FICare model was established in Canada to enable parents to become active caregivers for their infant, while participating as a member of the NICU team $(6,7)$. During the program, parents receive educational training from health care professionals on how to care for their infant, and have access to peer-to-peer support from veteran parents with prior experience. Nurses are also provided with tools and educational support to facilitate the integration of parents into the center of care. FICare not only improves neonatal outcomes, including increased weight gain and breastfeeding, but also enhances the social well-being of parents by reducing parental stress and anxiety. However, many health care professionals remain skeptical about FICare. Reasons for restricting parental access have been anecdotally described, including concerns over infection control, the convenient management of patients by health care staff, and traditional beliefs. Consequently, changing the paradigm of NICU is a rigorous process that involves widespread commitment of health care professionals, shifts in organizational culture, and recognition of barriers that prevent the implementation of evolving clinical practices.

Given that uptake of FICare relies on the support of unit policies and the entire NICU professional care team, the success of FICare in China depends on its complete acceptance by medical staff working in the NICU. It was hypothesized that neonatologists and NICU nurses may have different attitudes towards FICare, and there must be various concerns for implementation FICare in NICUs in China. The objective of this study was to assess current attitudes and concerns of neonatologists and nurses towards the implementation of FICare in Chinese NICUs. We present the following article in accordance with the SURGE reporting checklist (available at http://dx.doi.org/10.21037/ tp-20-60).

\section{Methods}

\section{Design, setting, and study participants}

Using a before and after study design, a qualitative analysis was conducted to determine the perceptions and attitudes of medical professionals towards FICare in China. A total of 34 neonatologists and 94 NICU nurses from 5 tertiary NICUs in 3 cities of 3 provinces in China volunteered to participate. No financial reward will be given to the participants. Two out the 3 cities were the capital city of the province, in detail: Hubei Province Women and Children Hospital, Wuhan, Hubei; the Third Xiangya Hospital of Central South University, Changsha, Hunan; Southeast University Affiliated Xuzhou Hospital, Xuzhou, Jiangsu; Hunan Children's Hospital, Changsha, Hunan; Hunan Provincial People's Hospital, Changsha, Hunan. All 3 cities were economically above the average level in China and with comparable economic conditions. All 5 NICUs had similar size and patient mix, which have no significant difference on space size, number of beds, number of nurses and physicians, nurse-to-bed ratio, nurse-to-physician ratio, number of preterm infants admitted per year, and average total hospital stay of preterm infants as described in the published FICare protocol (3). All 5 NIUCs were accredited as Grade A Level III NICUs authorized by the Health Administration of China. Responses to questions regarding various opinions and expectations of FICare were gathered from structured interviews before and after delivery of a getting buy-in education program on FICare. Individual concerns to implementation of FICare in NICUs in China were also encouraged to written down. A statistician will check the nonresponse error and completion ratio of the survey.

The study was conducted in accordance with the Declaration of Helsinki as revised in 2013. The study was approved by institutional Ethics Committee of Medical 
Research in Central South University (Ethic Approval Registration No. 2013-CSU-Ethic S123) and informed consent was taken from all the participants.

\section{Study steps}

A self-developed questionnaire, which was developed after group discussion by a group of NICU senior attending physicians, was used (see additional attachment for the details of the questionnaire). The survey was conducted in 2014 as part of the starting step of NICU FICare program in China (3). The detailed timeframe was: (I) from January 1 st to March 31, 2014, the questionnaire, the getting buyin education design and study registration was completed. (II) By the end of April 31, the initial materials and schedule of each participating NICU was completed. (III) From May 1 st to June 30 , the education program and surveys were completed. In order to avoid possible bias, the surveys were completed in an anonymous way.

The study steps were: First, the enrolled neonatologists and NICU nurses were given FICare publications and other family-centered care reading materials. After the reading, a survey with 11 multiple choice questions (Survey 1) was completed on the necessity and feasibility of FICare in China, and the potential of FICare to increase breastfeeding rates, shorten total hospital stay, and improve doctorpatient relationships, interest in joining a FICare program, the challenges and barriers of FICare implementation, and the potential negative outcomes associated with FICare. Then, a 4-hour presentation as getting buy-in session describing the interventions and outcomes of FICare was delivered, which included a description of the pilot study of FICare in Canada $(6,7)$ and the pilot implementation of FICare in China (3). This was provided by an educational nurse and a previous NICU fellow from Mount Sinai Hospital, Toronto, ON, Canada, where the pilot study was conducted. Following this session, the participants were invited to have a one-hour group discussion of FICare, whereby participants were encouraged to ask questions regarding their specific concerns. Thirdly, the participants were invited to complete the same survey (Survey 2) as they did before. Finally, the data was collected and analyzed by the education team.

\section{Statistical analysis}

The study population was summarized descriptively. It was considered as a non-response error if there were only 5 or less questions answered (more than half of the survey unanswered) on the survey or the survey was not sent back. The non-response error rate was calculated as '(number of non-response error/total number of participants) $\times 100 \%$ '. It was considered as a partial complete survey if there were 1-3 questions answered (less than one-third of the survey un-answered). It was considered as a complete survey if there were more than 8 questions answered. The response rate was calculated as '(number of partial and full completion/total number of participants) $\times 100 \%$ '. Among the 10 questions Difference in attitude toward FICare was compared between neonatologists and NICU nurses, using the Chi-square or Fisher exact test, as appropriate. Data management and statistical analyses were performed using SPSS 20.0 (USA). A significant level of $\mathrm{P}<0.05$ was used without adjustment for multiple comparisons.

\section{Results}

All participants (34 neonatologists and 94 NICU nurses) completed the education programs and answered all questions on the questionnaire (both the completion rate and the response rate were $100 \%$ ), indicating fairly good reliability and validity of the questionnaire.

In survey 1 (Table 1), responses between neonatologists and NICU nurses on the necessity and feasibility of implementing FICare in China, and its ability to shorten total hospital stay and improve doctor-patient relationships were similar. The willingness to adopt the FICare model was also comparable in both groups, as the majority expressed an interest to join FICare $(82.4 \%$ neonatologists vs. $83.0 \%$ nurses). However, opinions on whether FICare would promote breastfeeding in the NICU were mixed, as a significantly higher proportion of neonatologists responded positively comparing to nurses $(47.1 \% v s$. $19.1 \%$, respectively). Many participants in both groups also remained unsure whether FICare could help shorten the total hospital stay or improve doctor-patient relationships.

The top three barriers in applying the FICare model to current practice were identified by medical staff as: (I) insufficient space in NICU wards $(64.7 \%$ neonatologists vs. $61.7 \%$ NICU nurses), (II) lack of human resources (47.1\% neonatologists vs. $48.9 \%$ NICU nurses), and (III) inadequate funding to support FICare $(35.3 \%$ neonatologists vs. 27.7\% NICU nurses). Furthermore, the top three negative outcomes that would result from FICare implementation were perceived as: (I) an increase in hospital infections (70.1\% neonatologists vs. 68.1\% NICU nurses), 
Table 1 Attitude differences between neonatologists and NICU nurses to FICare

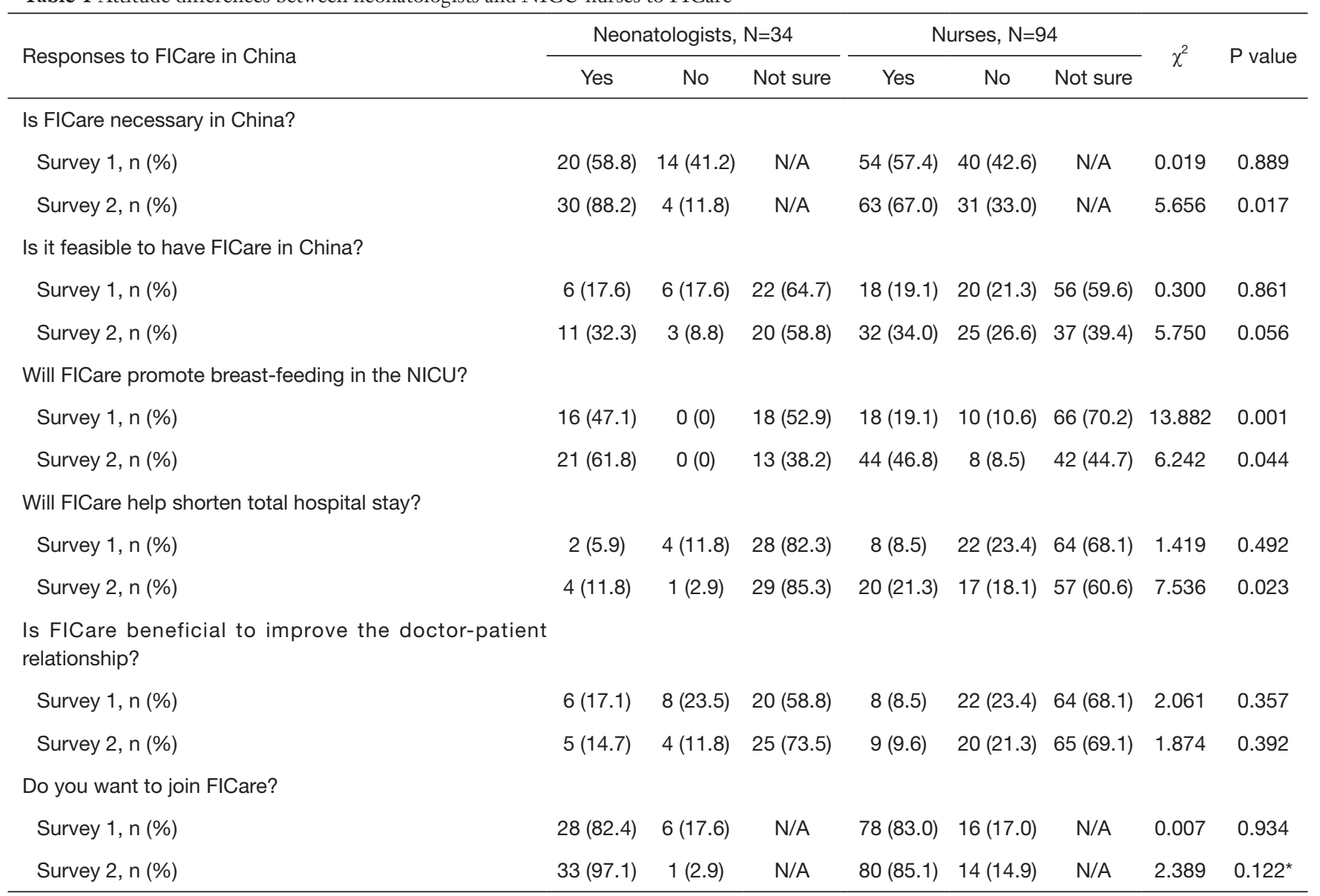

${ }^{*}$, correction for continuity. NICU, neonatal intensive care unit; FICare, family integrated care.

(II) a higher number of conflicts and complaints between medical staff and parents (64.7\% neonatologists vs. 51.1\% NICU nurses), and (III) parental non-cooperation (52.9\% neonatologists vs. $36.2 \%$ NICU nurses).

In survey 2 (Table 1), there was a significantly higher proportion of neonatologists considered FICare to be necessary, feasible, able to promote breast-feeding in the NICU and decrease total hospital stay. However, most remained unconvinced that FICare would be beneficial to the doctor-patient relationship.

Similar to above, the perceived barriers of FICare implementation remained unchanged after the education course, including insufficient space in the NICU ward (48.7\% neonatologists $v s$. 50\% NICU nurses), lack of human resources $(51.3 \%$ neonatologists vs. $56.3 \%$ NICU nurses), and inadequate funding to support FICare (25.6\% neonatologists $v s .32 .3 \%$ NICU nurses). In contrast, the top negative outcome believed to result from adopting the FICare model shifted from an increase in hospital infection to more conflicts and complaints between medical staff and parents (61.5\% neonatologists vs. $76 \%$ NICU nurses). Specifically, the percentage of medical staff that considered hospital infection to be the main concern decreased from $70.1 \%$ to $61.5 \%$ among neonatologists, and from $68.1 \%$ to $53.1 \%$ among NICU nurses. Meanwhile, $43.6 \%$ neonatologists and $38.5 \%$ NICU nurses had concerns regarding the non-cooperation of parents during FICare implementation.

\section{Discussion}

FICare adheres to the concepts and theories of familycentered care, which is broadly defined as an approach that promotes mutually beneficial partnerships between 
parents and healthcare professionals in the care of the infant to produce optimal outcomes (8). Thus far, parents have continuously been denied access to NICUs in China, and therefore do not participate in the care of their newborns during the NICU stay. The present study is the first to address the attitude and concerns of neonatologists and NICU nurses towards the FICare model, while to some extent assessing the impact of getting buy-in education program on producing an attitude shift in Chinese medical professionals. Overall, our findings suggest that attitude to FICare in NICUs in China is different while the concerns to FICare are similar among neonatologist and NICU nurses. The getting buy-in education program did have a discernable effect on changing the views of medical professionals on FICare. Results of this study can be used to inform the development of future educational curriculums and tools designed to stimulate interest in the FICare model.

In the current study, a high percentage of medical staff considered the FICare intervention to be unnecessary in China. Unfortunately, this is also an indication that several health care workers do not support the idea of FICare in Chinese NICUs, possibly due to the perceived risk of increased hospital infections. This was the largest concern identified by neonatologists and neonatal nurses despite a lack of empirical evidence. Additional concerns, which include the non-cooperation of Chinese parents with medical staff and a higher number of complaints, may also be key factors of resistance, as many argue that: (I) excluding parents from the ward allows doctors and nurses to consider their working environment to be purely medical, simple and convenient; (II) doctors and nurses save time by not having to persuade the mother to quit confinement in childbirth, which is a strong Chinese culture and tradition; and (III) the current socio-medical environment in China is rather tense $(9,10)$.

Our study revealed that the FICare getting buy-in education program was more successful in highlighting the necessity of FICare among neonatologists in comparison to NICU nurses (88.2\% versus $67.0 \%)$. The largest impact of the program on nurses' attitudes were on the feasibility of implementing FICare in China, it's potential to promote breastfeeding in the NICU and decrease total hospital stay. Meanwhile, the percentage of neonatologists and NICU nurses concerned about hospital infections both decreased after the education program. While it is difficult to determine why getting buy-in education program did not have a similar impact on doctors and NICU nurses on all aspects of FICare, it could be speculated that the limited sample size of the present study and a lack of strong, engaging material within the education program may have been key factors. Moreover, differences between physicians and nurses in terms of experiences in education may have contributed to a difference in attitudes, as clinicians may be more familiar with family integration. Similar exercises in Ireland, have shown an association between education and nurses' perceptions of family-centered care (11). In contrast, a previous survey of nurses in a single pediatric tertiary care center in Nova Scotia, Canada, revealed that perceptions about the necessary components of family-centered care varied with age and clinical position, while education and experience were not influencers (12). Other groups have reported that the presence of developmental care specialists in the unit can also influence neonatal nursing perceptions of family-centered car3 (13).

It is evident that a single exposure to the getting buyin session would not be sufficient to alter the opinions of medical professionals, particularly those unfamiliar with the model. Perhaps extending the 4-hour getting buy-in lectures and group discussion time, in addition to allowing medical professionals to periodically repeat the education program, may result in a higher reception of the model. Moreover, observatory site visits to units already practicing FICare may further help engage professionals. Another alternative approach may be to showcase other attainable outcomes of FICare, such as decreased parental stress, increased weight gain and parental confidence at discharge, and other aspects of FICare philosophy. Finally, an understanding of barriers perceived by medical staff prior to the course may help guide discussions on mitigation strategies to overcome such challenges, and reduce the existing resistance towards change.

The limitations of this study include its reliance on data collected from medical staff working in five neonatal units with an above-average economic level. There are 28 provinces in China with different economic and health development, which has a considerable impact on outcome of neonates in NICU. All 3 provinces included in this study were economically above the average level in China. All 5 NICUs included in this study had similar size, patient mix, and all were at the same NICU Level authorized by the Health Administration of China. Therefore, we consider them are of comparable economic conditions and NICU capacity. But there was a significant difference between the number of nurses and the number of doctors surveyed. Therefore, results and opinions expressed in this study 
may not be generalizable to other regions and provinces in China. We also did not collect any demographic characteristics from individual participants, and could not assess whether responses were gender, training, or education-specific. These factors may be important when designing future educational tools. Lastly, given that participation in the study was voluntary, it is likely that medical staff were already receptive to the FICare model, thereby introducing subject bias. This is evident in our preeducation analysis, as a very high percentage $(>80 \%)$ of respondents were inclined to join FICare, even though the majority were skeptical about its necessity, feasibility and deliverables.

\section{Conclusions}

The successful implementation of FICare depends on the well accepting attitude of NICU staffs and nurses. The results of this study revealed different attitude to FICare among them. Both NICU staffs and nurses have similar concerns to FICare, which, to some extent, raised the importance of getting buy-in activities, including enhanced staff education and training courses, for promoting FICare in China.

\section{Acknowledgments}

The authors thank Ms. Ruth Warr from the MaternalInfant Care Research Centre in Toronto for the editorial support, and Mr. Philip Ye from the Maternal-Infant Care Research Centre in Toronto for the statistical guidance. The authors also thank Ms. Yanjun Tan and Ms. Li He for their help in uploading the raw data into the database.

Funding: This study was supported by the Special Fund of the Pediatric Medical Coordinated Development Center of Beijing Hospitals Authority (No. XTCX201816, issued to Dr. Mingyan Hei). This study was also supported in partial by Canadian Institutes of Health Research Team Grant (CTP 87517, issued to Dr. Mingyan Hei) in MaternalInfant Care from Canada. These funders had no role in the study design, collection, analysis or interpretation of the data, writing the manuscript, or the decision to submit the paper for publication.

\section{Footnote}

Reporting Checklist: We present the following article in accordance with the SURGE reporting checklist. Available at http://dx.doi.org/10.21037/tp-20-60

Data Sharing Statement: Available at http://dx.doi. org/10.21037/tp-20-60

Peer Review File: Available at http://dx.doi.org/10.21037/ tp-20-60

Conflicts of Interest: All authors have completed the ICMJE uniform disclosure form (available at http://dx.doi. org/10.21037/tp-20-60). The authors have no conflicts of interest to declare.

Ethics Statement: The authors are accountable for all aspects of the work in ensuring that questions related to the accuracy or integrity of any part of the work are appropriately investigated and resolved. The study was conducted in accordance with the Declaration of Helsinki as revised in 2013. The study was approved by institutional Ethics Committee of Medical Research in Central South University (Ethic Approval Registration No. 2013-CSUEthic S123) and informed consent was taken from all the participants.

Open Access Statement: This is an Open Access article distributed in accordance with the Creative Commons Attribution-NonCommercial-NoDerivs 4.0 International License (CC BY-NC-ND 4.0), which permits the noncommercial replication and distribution of the article with the strict proviso that no changes or edits are made and the original work is properly cited (including links to both the formal publication through the relevant DOI and the license). See: https://creativecommons.org/licenses/by-nc-nd/4.0/.

\section{References}

1. Kong X, Xu F, Wu R, et al. Neonatal mortality and morbidity among infants between 24 to 31 complete weeks: a multicenter survey in China from 2013 to 2014. BMC Pediatr 2016;16:174.

2. Wei KL, Yang YJ, Yao YJ, et al. Epidemiologic survey on hospitalized neonates in China. Transl Pediatr 2012;1:15-22.

3. Hei $M$, Gao X, Nong $S$, et al. Is family integrated care in neonatal intensive care units feasible and good for preterm infants in China: study protocol for a cluster randomized 
controlled trial. Trials 2016;17:22.

4. Li XY, Lee S, Yu HF, et al. Breaking down barriers: enabling care-by-parent in neonatal intensive care units in China. World J Pediatr 2017;13:144-51.

5. Wu S, Zhang L, Zhou X. Analysis of maternal psychological status of NICU admitted newborns and the nursing strategies. Fujian Med J 2011;33:144.

6. O'Brien K, Bracht M, Robson K, et al. Evaluation of the Family Integrated Care model of neonatal intensive care: a cluster randomized controlled trial in Canada and Australia. BMC Pediatr 2015;15:210.

7. O'Brien K, Robson K, Bracht M, et al. Effectiveness of Family Integrated Care in neonatal intensive care units on infant and parent outcomes: a multicentre, multinational, cluster-randomised controlled trial. Lancet Child Adolesc Health 2018;2:245-54.

Cite this article as: $\mathrm{Xiang} X, \mathrm{Xia} S$, Zhu $\mathrm{X}$, Gao $\mathrm{X}$, Gao X, Zhang A, Lee SK, Hei M. Attitudes and concerns of neonatologists and nurses to family-integrated-care in neonatal intensive care units in China. Transl Pediatr 2020;9(5):603-609. doi: 10.21037/tp-20-60
8. Smith L, Coleman V, Bradshaw ME. Family-centered care: concept, theory and practice. Palgrave: Houndmills, 2002.

9. Huang SL, Ding XY. Violence against Chinese health-care workers. Lancet 2011;377:1747.

10. Jie L. New generations of Chinese doctors face crisis. Lancet 2012;379:1878.

11. Coyne I, Murphy M, Costello T, et al. A survey of nurses' practices and perceptions of family-centered care in Ireland. J Fam Nurs 2013;19:469-88.

12. Bruce B, Ritchie J. Nurses' practices and perceptions of family-centered care. J Pediatr Nurs 1997;12:214-22.

13. Hendricks-Muñoz KD, Louie M, Li Y, et al. Factors that influence neonatal nursing perceptions of family-centered care and developmental care practices. Am J Perinatol 2010;27:193-200. 